\title{
S-matrix poles for chaotic quantum systems as eigenvalues of complex symmetric random matrices: from isolated to overlapping resonances
}

\author{
Hans-Jürgen Sommers§, Yan V. Fyodorov§, $\boldsymbol{\uparrow}$, and Mikhail Titov $\boldsymbol{\uparrow}$ \\ §Fachbereich Physik, Universität-GH Essen, D-45117 Essen, Germany \\ ๆ Petersburg Nuclear Physics Institute, Gatchina 188350, Russia
}

(June 29, 2018)

\begin{abstract}
We study complex eigenvalues of large $N \times N$ symmetric random matrices of the form $\mathcal{H}=\hat{H}-i \hat{\Gamma}$, where both $\hat{H}$ and $\hat{\Gamma}$ are real symmetric, $\hat{H}$ is random Gaussian and $\hat{\Gamma}$ is such that $N \operatorname{Tr} \hat{\Gamma}_{2}^{2} \sim \operatorname{Tr} \hat{H}_{1}^{2}$ when $N \rightarrow \infty$. When $\hat{\Gamma} \geq 0$ the model can be used to describe the universal statistics of S-matrix poles (resonances) in the complex energy plane. We derive the ensuing distribution of the resonance widths which generalizes the well-known $\chi^{2}$ distribution to the case of overlapping resonances. We also consider a different class of "almost real" matrices when $\hat{\Gamma}$ is random and uncorrelated with $\hat{H}$.
\end{abstract}

As is well-known, S-matrix poles or resonances can be looked at as complex eigenvalues of an effective non-Hermitian Hamiltonian $\mathcal{H}$ emerging after the so-called complex rotation was applied to the original Hermitian Hamiltonian of the system. The method [1] allows one to extract resonance positions $E_{k}$ and widths $\Gamma_{k}$ directly, without expensive evaluation of the energy-dependent $S$ - matrix elements. The search of resonances typically amounts to diagonalizing large complex symmetric matrices representing the effective Hamilonian in a suitable basis on a finite grid. Using this method, patterns consisting of hundreds of individual resonances were extracted recently for realistic models in atomic and molecular physics [2,37. Frequently, for high enough excitation energies the S-matrix poles are placed irregularly in the complex plane, forming a structure of a "chaotic jumble" [3], the situation calling for a statistical description. As another important development it is appropriate to mention a powerful numerical algorithm that was proposed to extract resonance positions and widths with a high accuracy from the experimentally accessible time correlation function of finite duration [4. All these facts make the problem of statistical description of resonances to be an important and opportune task.

At the same time, the statistics of highly excited bound states of closed quantum systems of quite different microscopic nature is known to be system-independent (universal), provided the corresponding classical counterparts demonstrate a well-developed chaotic motion [5]. Moreover, the spectral correlations turn out to be exactly the same as provided by the theory of large random matrices on the scale determined by a typical separation $\Delta$ between neighbouring eigenvalues. Microscopic justifications of the use of random matrices for describing the universal properties of quantum chaotic systems have been provided by several groups recently, based both on traditional semiclassical periodic orbit expansions [6, 7] and on advanced field-theoretical methods [8,9]. It is natural to try to develop a description of universal statistical properties of resonances using the same ideas.

The methods to adjust random matrix description to the case of open chaotic systems are well known since the pioneering paper [10] and described in much detail in [11]. The starting point of this approach is the representation of the scattering matrix $\hat{S}$ in terms of an effective non-Hermitian Hamiltonian $\mathcal{H}_{e f f}=\hat{H}-i \hat{\Gamma}$. Here the Hermitian Hamiltonian $\hat{H}$ describes the closed counterpart of the open system whereas the anti-Hermitian part $i \hat{\Gamma}$ arises due to coupling to open scattering channels. It has to be chosen in the form ensuring the unitarity of the scattering matrix. As a 
result all eigenvalues of $\hat{\Gamma}$ turn out to be non-negative (as is also required by causality), the number of strictly positive eigenvalues being just the number $M$ of open channels.

It is natural to expect, that statistical properties of resonances are inherited from the corresponding random matrix universality of levels of closed systems. Remembering the discussion above, we expect them to be universal on the energy scales in the complex plane comparable with the mean level spacing for the closed system $\Delta$. In contrast, properties on a much larger scale must be highly system-specific.

A natural way to incorporate the random matrix description of the quantum chaotic system is to replace $\hat{H}$ by a large $N \times N$ random matrix of appropriate symmetry. Namely, chaotic systems with preserved time-reversal invariance (TRI) should be described by matrices $H_{i j}$ which are real symmetric. Such matrices form the Gaussian Orthogonal Ensemble (GOE), whereas for systems with broken TRI one uses complex Hermitian matrices from the Gaussian Unitary Ensemble (GUE) [5]. As to the matrix $\hat{\Gamma}$ it is determined by the coupling of the chaotic system to open channels and can be considered as a fixed given one of the same dimension.

In general, the two matrices $\hat{H}$ and $\hat{\Gamma}$ do not commmute, the fact making the analysis of complex eigenvalues of $\mathcal{H}$ to be a rather non-trivial problem. In the simplest case one can treat the antiHermitian part as a small perturbation to the Hermitian one. This is justified for the regime of isolated resonances, i.e. when typical resonance widths are much smaller than the mean separation $\Delta$ between the positions of neighbouring resonances. Assuming for simplicity that all scattering channels (hence, the eigenvalues of $\hat{\Gamma}$ ) are statistically equivalent, one arrives at the so-called $\chi^{2}$ distribution of the resonance widths. It was introduced originally many years ago by Porter and Thomas and since then experimentally observed in many physical systems, see some references in [12]. The corresponding perturbative expression is known also for the case of non-equivalent scattering channels 13.

Until quite recently relatively little was known about resonance statistics non-perturbatively. Among the known facts were the joint probability density of all resonances (however, not its moments) for the systems with only one open channel $M=1$ [14], and the mean density of complex eigenvalues for very many open channels $M \sim N \gg 1$ [15]. The most essential progress was achieved recently [16,11] by employing a powerful field-theoretical method known as Efetov's supersymmetric non-linear $\sigma$-model [17]. It turns out to be possible to find explicitly the mean density of the resonances for systems with broken time-reversal invariance and to put forward a wellgrounded conjecture on the higher correlation functions in the complex plane [18]. Unfortunately, the most interesting and practically important case of the systems with preserved time-reversal invariance proved to be less accessible analytically (for numerics see 19]) and no results of such generality were obtained for such systems so far.

The main goal of the present paper is to fill in this gap partly by presenting the exact nonpertubative distribution of the resonance widths for quantum chaotic systems with preserved timereversal invariance.

It is necessary to menion that random matrices (more generally, random linear operators) with complex eigenvalues emerged recently in many other physical contexts. A few recent examples include the description of thermal motion of an isolated vortex in disordered type-II superconductors with columnar defects, the problem of a classical diffusing particle advected by a stationary random velocity field and attempts to understand the universal features of chiral symmetry breaking in Quantum Chromodynamics (a more detailed discussion and the relevant references can be found in the papers mentioned below). At the same time, our knowledge of statistical properties of random non-selfadjoint matrices is quite scarce and incomplete. This fact recently stimulated efforts of different groups to improve our understanding in this direction 20 24]. In particular, the 
existence of a nontrivial regime of weak non-Hermiticity was recognized in the work [22], see more detailed discussion in 24]. The guiding idea to realize the existence of such a regime comes from the experience with resonances [16. Guided by that example one guesses that a new regime occurs when the imaginary part of typical eigenvalues is comparable with the mean separation between neighboring eigenvalues along the real axis.

One can use again the supersymmetry approach to obtain the mean density of complex eigenvalues in the regime of weak non-Hermiticity for matrices with independent elements 22,24. The density turned out to be described by a formula containing as two opposite limiting cases both the Wigner semicircular density of real eigenvalues of the Hermitian matrices and the uniform density of complex eigenvalues discovered for usual non-Hermitian random matrices already by Ginibre [25], in much details addressed by Girko [26] and other authors [27. In his insightful paper Efetov [28] managed to derive the density of complex eigenvalues for a related, but different set of (almostsymmetric) real random matrices. His result was generalized in [18]. Actually, the non-Hermitian matrices considered in 22] and 28] are just two limiting cases of a general three-parameter family of "weakly non-Hermitian" ensembles [24] with independent entries. Apart from these two cases, the mentioned three-parameter family contains one more important particular case corresponding to an ensemble of complex symmetric random matrices. Such matrices are natural to call the "almost-real" ones. The second goal of the present paper is to provide the eigenvalue density for this particular ensemble.

As a starting point of our investigation we use the representations of the mean density $\rho(X, Y)$ of eigenvalues $Z=X+i Y$ with a given imaginary part $Y$ derived by us earlier [24,29]. Let us first consider the case relevant to the chaotic scattering. Due to rotational invariance of the GOE matrices the result can be dependent only on the set of positive eigenvalues $\gamma_{1}, \gamma_{2}, \ldots, \gamma_{M}$ of the matrix $\hat{\Gamma}$. Correspondingly, the density $\rho_{X}(y) \equiv \rho(X, Y) \Delta^{2}(X) / \pi$ of scaled resonance widths $y=\frac{\pi Y}{\Delta}$ (measured in units of the local mean level spacing $\Delta(X)=1 /(N \nu(X))$ of the closed system, with $\nu(X)=\pi^{-1}\left(1-X^{2} / 4\right)^{1 / 2}$ being the semicircular density of real eigenvalues) for those resonances whose positions are within the narrow window around the point $X$ of the spectrum is given by:

$\left\langle\rho_{X}(y)\right\rangle=\frac{1}{16} \int d \mu(\hat{Q}) \operatorname{Str}\left(\hat{\sigma}_{\tau}^{(F)} \hat{Q}\right) \operatorname{Str}\left(\hat{\sigma}_{\tau} \hat{Q}\right) \prod_{a=1}^{M} \operatorname{Sdet}^{-1 / 4}\left[1-\frac{i}{2 g_{a}}\left\{\hat{Q}, \hat{\sigma}_{\tau}\right\}\right] \exp \frac{i}{2} y \operatorname{Str}\left(\hat{\sigma}_{\tau} \hat{Q}\right)$

We introduced here the parameters $g_{a} \in[1, \infty)$ characterizing the coupling of a system to open channels which are expressed in terms of the eigenvalues $\gamma_{a}$ as $g_{a}=\frac{1}{2 \pi \nu(X)}\left(\gamma_{a}+\gamma_{a}^{-1}\right)$. The integration above goes over the set of $8 \times 8$ supermatrices $\hat{Q}$ satisfying the constraint $\hat{Q}^{2}=-1$ and some symmetry requirements, which can be conveniently resolved if one parametrizes the manifold as: $\hat{Q}=-i Z \Lambda Z^{-1}$, with $\Lambda=\operatorname{diag}(\mathbf{1},-\mathbf{1})$, each $\mathbf{1}$ representing $4 \times 4$ unity matrix. The symbols $S t r, S d e t$ stand for the graded trace and the graded determinant, correspondingly, and $\left\{\hat{Q}, \hat{\sigma}_{\tau}\right\}=\hat{Q} \hat{\sigma}_{\tau}+\hat{\sigma}_{\tau} \hat{Q}$ stands for the anticommutator. Other $8 \times 8$ supermatrices entering this expressions are as follows:

$$
\hat{\sigma}_{\tau}^{(F)}=\left(\begin{array}{cc}
\hat{0}_{4} & \hat{\tau}_{3}^{(F)} \\
\hat{\tau}_{3}^{(F)} & \hat{0}_{4}
\end{array}\right) ; \quad \hat{\sigma}_{\tau}=\left(\begin{array}{cc}
0 & \hat{\tau}_{3} \\
\hat{\tau}_{3} & 0
\end{array}\right)
$$

and $\hat{\tau}_{3} ;, \hat{\tau}_{3}^{(F)}$ are $4 \times 4$ diagonal supermatrices: $\hat{\tau}_{3}=\operatorname{diag}\{\hat{\tau}, \hat{\tau}\} ; \hat{\tau}_{3}^{(F)}=\operatorname{diag}\left\{\hat{0}_{2}, \hat{\tau}\right\}$. with $\hat{\tau}=$ $\operatorname{diag}(1,-1)$.

For the case of the matrix $\hat{\Gamma}$ being real symmetric with entries independently fluctuating around zero and normalized in such a way that $\lim _{N \rightarrow \infty} \operatorname{Tr} \hat{\Gamma}_{2}^{2}<\infty$ (the condition of being "almost 
symmetric") the corresponding expression for the mean density of eigenvalues is obtained from Eq.(11) by replacing the product of superdeterminants in the integrand with the exponent:

$$
\exp \left\{-\frac{v^{2}}{4} \operatorname{Str}\left(\hat{\sigma}_{\tau} \hat{Q}\right)^{2}\right\}
$$

where $v^{2}=(\pi \nu)^{2} \lim _{N \rightarrow \infty} \operatorname{Tr} \hat{\Gamma}_{2}^{2}$, see the derivation in [24].

The starting formulas are actually valid both for real symmetric and complex Hermitian matrices $H$. To extract the explicit form of the distribution function one still has to perform the integration over the manifold of the supermatrices $Q$. Still, it is rather difficult calculation due to a cumbersome structure of that manifold, and the success is mainly determined by finding an appropriate parametrization. For the simplest case of matrices $\hat{H}$ being complex Hermitian the supermatrices $Q$ become block-diagonal due to additional constraints and the calculation, although cumbersome, can be still done successfully [16, 11,22] in the "standard" Efetov parametrization [17]. For the case of real symmetric matrices with resulting full $8 \times 8$ structure of $Q$ this is no longer the case. Fortunately, we are able to parametrize the matrices $Z$ as $Z_{1} Z_{2}$ in such a way, that $Z_{1}$ commutes with $\hat{\sigma}_{\tau}$, thus dropping out from the integrand. We would like to stress that although we enjoyed some insights from the recent paper on real asymmetric matrices 28, our actual parametrization is quite different from that used in 28. A derivation of the explicit form of such a parametrization and especially the integration measure turn out to be very lengthy and will be presented in details elsewhere. Here we would like to present only the result of integrating out the Grassmannian degrees of freedom.

$$
\left\langle\rho_{X}(y)\right\rangle=\frac{-1}{16 \pi} \frac{\partial}{\partial y} \int_{-1}^{1} d \lambda f_{s}^{-2}(\lambda) \int_{-\infty}^{\infty} d \lambda_{1} f_{s}\left(i \lambda_{1}\right) \int_{\lambda_{1}}^{\infty} d \lambda_{2} f_{s}\left(i \lambda_{2}\right) \frac{\left(\lambda_{2}-\lambda_{1}\right)\left(2 \lambda-i \lambda_{1}-i \lambda_{2}\right)}{\left(\lambda-i \lambda_{1}\right)^{2}\left(\lambda-i \lambda_{2}\right)^{2}}
$$

where we use the convention $s=r$ for the case of resonance statistics and $s=R$ for that case when the antihermitian part $\hat{\Gamma}$ is a random matrix. Correspondingly, we defined

$$
f_{s}(\lambda)=\frac{\exp \lambda y}{\left(1-\lambda^{2}\right)^{1 / 2}} \times \begin{cases}\frac{1}{\prod_{a=1}^{M}\left(g_{a}-\lambda\right)^{1 / 2}} & \text { for resonances, } s=r \\ \exp \left(v^{2} \lambda^{2}\right) & \text { for a random matrix, } s=R\end{cases}
$$

The following important comment is appropriate here. The integrals over $\lambda_{1}, \lambda_{2}$ as they stand in Eq.(2) are not well-defined when taken literally along the real axis due to a singularity at the points $\lambda_{1}=\lambda=0 ; \lambda_{2}=\lambda=0$. This difficulty can be traced back to a singularity of the transformation arising from diagonalization of some supermatrices when coming to a new parametrization mentioned above. A more accurate consideration shows that the integration over $\lambda_{1}, \lambda_{2}$ should be understood in the sense of principal value. To be precise, the correct expression is equal to the half-sum of two integrals with integration contours encircling the singular point $\lambda_{1}=-i \lambda ; \lambda_{2}=-i \lambda$ from above and from below.

The expression Eq.(2) is exact, but still is not very convenient for evaluation. Fortunately, for the physically motivated case of resonances one can deform the contour in such a way, that it goes along the cut $\lambda_{1,2}=-i p, p \in[1, \infty)$ for negative values of $y$ and along a similar cut $\lambda_{1,2}=i p, p \in[1, \infty)$ for $y>0$. At the same time, all the singularities $\lambda_{1}=-i g_{a}, a=1, \ldots, M$ belong to the cut in the lower half-plane. This analytic structure of the integrand ensures vanishing of the density for $y>0$ as expected, whereas for $y<0$ one obtains:

$$
\begin{gathered}
\rho_{X}(y<0)=\frac{1}{4 \pi} \frac{\partial^{2}}{\partial y^{2}} \int_{-1}^{1} d \lambda\left(1-\lambda^{2}\right) \int_{1}^{\infty} \frac{d p_{1}}{\left(p_{1}^{2}-1\right)^{1 / 2}} \int_{1}^{p_{1}} \frac{d p_{2}}{\left(p_{2}^{2}-1\right)^{1 / 2}} \frac{\left(p_{1}-p_{2}\right) e^{+y\left(p_{1}+p_{2}-2 \lambda\right)}}{\left(\lambda-p_{1}\right)^{2}\left(\lambda-p_{2}\right)^{2}} \\
\times \chi_{1}\left(p_{2}\right) \chi_{2}\left(p_{1}\right) \prod_{a=1}^{M} \frac{\left(g_{a}-\lambda\right)}{\left(\left|g_{a}-p_{1}\right|\left|g_{a}-p_{2}\right|\right)^{1 / 2}}
\end{gathered}
$$


where the functions $\chi_{1,2}(p)$ are defined as follows:

$$
\chi_{1}(p)=\left\{\begin{array}{l}
1 \text { for } g_{4 k} \leq p \leq g_{4 k+1} \\
-1 \text { for } g_{4 k+2} \leq p \leq g_{4 k+3} \\
0 \text { otherwise }
\end{array} \quad \chi_{2}(p)=\left\{\begin{array}{l}
1 \text { for } g_{4 k+1} \leq p \leq g_{4 k+2} \\
-1 \text { for } g_{4 k+3} \leq p \leq g_{4 k+4} \\
0 \text { otherwise }
\end{array}\right.\right.
$$

where $k=0,1, \ldots,[M / 4]$ and we used a convention: $1 \equiv g_{0} \leq g_{1} \leq \ldots \leq g_{M}<g_{M+1}=\infty$.

The formulas Eqs.(3) provide us with the most general explicit analytical expression for the density of complex resonances for a chaotic quantum system with preserved time-reversal invariance. As such they constitute the main result of the present paper.

We see, that in general these formulas are still quite cumbersome. One can infer from them various known limiting cases considered earlier. For example, we can consider the case of weak coupling to continuum when all $g_{a} \gg 1$. By noticing that the integration over $p_{2}$ in Eq.(3) is dominated in this case by $p_{2} \sim 1$, whereas $p_{1} \geq g_{1}>>1, \lambda, p_{2}$ and taking into account also that typical values of $y$ are of the order of $g_{1}^{-1}<<1$ one can evaluate the integrals over $p_{2}, \lambda$ to the leading order. The result is:

$$
\rho_{X}(y<0)=\frac{1}{\pi} \prod_{a} g_{a}^{1 / 2} \int_{1}^{\infty} \frac{d p_{1}}{\prod_{a}\left|g_{a}-p_{1}\right|^{1 / 2}} e^{y p_{1}} \chi_{2}\left(p_{1}\right)
$$

which coincides with the distribution derived recently by Alhassid and Lewenkopf 13] by a perturbative treatment, up to alternating signs in the factor $\chi_{2}(p)$ which is an apparent misprint in their paper.

Relying on earlier experience with resonance statistics in systems with broken time-reversal invariance 16, 11, , one might expect that the distribution simplifies drastically for the case of statistically equivalent channels: $g \equiv g_{1}=g_{2}=\ldots=g_{M}$. Surprisingly, this seems to be not the case for the present model. Actually, we find it difficult to perform such a limit explicitly for arbitrary number of open channels, and even for $M=4$ the result turns out to be quite cumbersome. Below we present the formula for one and two open channels $M=1,2$ :

$$
\rho_{X}(y<0)=\frac{1}{4 \pi} \frac{\partial^{2}}{\partial y^{2}} \int_{-1}^{1} d \lambda\left(1-\lambda^{2}\right) e^{-2 \lambda y} F_{M}(\lambda, y)
$$

where for $M=1$ we found

$$
F_{1}=(g-\lambda) \int_{g}^{\infty} d p_{1} \frac{e^{y p_{1}}}{\left(p_{1}^{2}-1\right)^{1 / 2}\left(\lambda-p_{1}\right)^{2}\left(p_{1}-g\right)^{1 / 2}} \int_{1}^{g} d p_{2} \frac{e^{y p_{2}}}{\left(p_{2}^{2}-1\right)^{1 / 2}} \frac{\left(p_{1}-p_{2}\right)}{\left(\lambda-p_{2}\right)^{2}\left(g-p_{2}\right)^{1 / 2}}
$$

and the case $M=2$ is the simplest one:

$$
F_{2}=\frac{\pi}{\left(g^{2}-1\right)^{1 / 2}} \int_{1}^{g} d p_{2} \frac{1}{\left(p_{2}^{2}-1\right)^{1 / 2}} \frac{e^{y\left(p_{2}+g\right)}}{\left(\lambda-p_{2}\right)^{2}}
$$

The figures Fig.1 and and Fig.2 show that the resulting expression favourably agree with the results of direct numericaldiagonalization of the corresponding complex matrices.

Despite the fact, that the general resonance widths distribution for the case of preserved TRS turns out to be much more complicated than for the case of broken TRS, both expressions share many important features. For example, evaluating the first moment of the distribution Eq.(3) for $M=2$ exactly, one arrives at the following expression for the mean resonance widths:

$$
\frac{\langle\Gamma\rangle}{\Delta}=-\frac{1}{\pi} \ln \frac{g-1}{g+1}
$$


This formula is well known in nuclear physics as Moldauer-Simonius relation and follows from the unitarity of the scattering matrix 30]. Actually, a simlar relation must be satisfied for an arbitrary number $M$ of open channels. It was indeed found to be the case for systems with broken TRS [11]. Unfortunately, it is quite difficult to verify the Moldauer-Simonius relation directly from the general distribution Eq.(3).

The logarithmic divergency at $g_{a}=1$ signals on the powerlaw tail behaviour $\rho_{X}(|y| \gg 1) \propto y^{-2}$ typical for this (a so-called "perfect coupling") case. It is instructive to perform the limit $g \rightarrow 1$ directly in Eq.(5) for $M=2$ and find:

$$
\rho_{X}(y<0)=\frac{1}{4 y^{3}}\left(2 y+1+(2 y-1) e^{4 y}\right)
$$

where such a behaviour is self-evident.

Actually, it is interesting to compare this expression with the $M=1$ case for systems with broken TRS [16]:

$$
\rho_{X}(y<0)=\frac{1}{2 y^{2}}\left(1+(2 y-1) e^{2 y}\right)
$$

The reason is that in the regime of isolated resonances $g \gg 1$ the M-channel system with broken TRS possesses exactly the same widths distribution as the $2 M$ channel system with unbroken TRS. A similar correspondence also holds in the limit of many open channels $M \gg 1$. Moreover, before the exact formula Eq. (7) was available, an attempt was made 31] to compare $M=1$ formula Eq.(8) valid for systems with broken TRS with numerically found resonance widths in $M=2$ chaotic TRS-preserving microwave cavity. The correspondence was reported to be quite satisfactory.

We see, that both formulas indeed give exactly the same behaviour for the resonances whose widths exceed the typical separation between them: $\rho_{X}(|y| \gg 1) \approx 1 / 2 y^{2}$. However, in the region of very narrow resonances the behaviour is slightly different: $\rho_{X}(y \rightarrow 0)=1$ for broken and $\rho(y \rightarrow$ $0)=4 / 3$ for unbroken TRS. This fact shows the limitations of the mentioned correspondence.

As to the random-perturbation case $(s=R$ in eq.(2) $)$, the presence of the Gaussian factors in the integrand of Eq.(2) prevents one from deforming the contour to the complex plane. At the moment, we are unable to simplify the expression further. However, one can still infer that in the perturbative case $v \ll 1$ the function $\rho_{X}(y)$ reduces to a simple Gaussian, whereas in the opposite limiting case $v \gg 1$ one indeed arrives at the "elliptic law" well-known to hold for strongly non-Hermitian random matrices [26,27.

The authors are much obliged to B.A.Khoruzhenko for useful communications. The work was supported by SFB 237 "Unordnung und grosse Fluktuationen" and in part by RFBR Grant No. 96-02-18037-a.

[1] E.Baslev and J.Combes, Commun.Math.Phys.. 22, 280 (1971); See also a recent review by N.Moiseev, to appear in Phys.Rep. (1998)

[2] J.Main and G.Wunner J.Phys.B: At.Mol., 27, 1994 (1994)

[3] R.Blümel,

Phys.Rev.E, 54, 5420 (1996); V.A.Mandelshtam and H.S.Taylor, J.Chem.Soc.Faraday.Trans. , 93 ,847 (1997) 
[4] V.A.Mandelshtam and H.S.Taylor, Phys.Rev.Lett., 78, 3274 (1997)

[5] O.Bohigas, in Chaos and Quantum Physics. Proceedings of the Les-Houches Summer School. Session LII, ed. by M.J.Giannoni et.al (North Holland, Amsterdam, 1991), p.91

[6] M.Berry, Proc.R.Soc.London, Ser.A 400, 229 (1985);

[7] E.Bogomolny and J.Keating, Phys.Rev.Lett 77, 1472 (1996)

[8] B.A.Muzykantsky and D.E.Khmelnisky JETP Lett. 62 , 76 (1995);

[9] A.Andreev, O. Agam, B. Altshuler and B.Simons . Phys.Rev.Lett., 76, 3947 (1996)

[10] J.J.M.Verbaarschot, H.A.Weidenmüller, M.R.Zirnbauer , Phys.Rep. v.129 (1985), 367

[11] Y.V.Fyodorov and H.-J.Sommers , J.Math. Phys., 38 , 1918 (1997)

[12] P.Gaspard in "Quantum Chaos", Proceedings of E.Fermi Summer School,1991 ed. by G.Casati et al. (North Holland, Amsterdam,1991), p.307

[13] Y.Alhassid and C.H.Lewenkopf Phys.Rev.Lett.,75 3922,(1995); W.H. Miller, R. Hernandez, C.B. Moore and W.H. Polik, J. Chem. Phys. 93, 5657 (1990).

[14] V.V.Sokolov and V.G.Zelevinsky Phys.Lett.B 202, 10 (1988); Nucl.Phys.A 504, 562 (1989); H.-J. Stöckmann and P.Seba, J.Phys.A:Math.Gen. 31, 3439 (1998)

[15] F.Haake, F.Izrailev, N.Lehmann, D.Saher, and H.-J.Sommers, Z.Phys.B 88, 359 (1992); N.Lehmann, D.Saher, V.V.Sokolov, and H.-J.Sommers, Nucl.Phys.A 582, 223 (1995);

[16] Fyodorov Y V and Sommers H-J Pis'ma ZhETF v.63 (1996),970; [JETP Letters v.63 (1996),1026]

[17] K.B. Efetov Supersymmetry in Disorder and Chaos (Cambridge University Press,1996 )

[18] Y.V. Fyodorov, M.Titov and H.-J. Sommers, e-preprint cond-mat/9802306, to appear in Phys.Rev.E

[19] T.Gorin, F.-M.Dittes, M.Müller, I.Rotter and T.H.Seligman , Phys.Rev.E, v.56 (1997), 2481

[20] M.A. Stephanov, Phys. Rev. Lett. 76, 4472 (1996); R.A.Janik et al., Phys. Rev. Lett. 77, 4876 (1996); M.A. Halasz, A.D., J.C.Osborn and J.J.M. Verbaarschot, Phys.Rev.D, v.56 (1997), 7059; R.A. Janik, M.Nowak,G.Papp and I.Zahed Nucl.Phys.B 501, 603 (1997); J. Feinberg and A. Zee, Nucl.Phys.B. 501,643(1997) and ibid 504, 579 (1997)

[21] B.A.Khoruzhenko, J.Phys.A 29, L165 (1996).

[22] Y.V. Fyodorov, B. Khoruzhenko and H.-J. Sommers, Physics Letters A 226, 46 (1997) and Phys.Rev.Lett. v. 79 (1997), 557

[23] M. Kus, F. Haake, D. Zaitsev and A.Huckleberry, J.Phys.A 30, 8635 ( 1997)

[24] Y.V. Fyodorov, B. Khoruzhenko and H.-J. Sommers, Ann.Inst. Henri Poincare, Physique Theorique v.66, $449(1998)$

[25] J. Ginibre, J. Math. Phys. 6, 440 (1965)

[26] V. Girko, Theor. Prob. Appl. 30, 677 (1986)

[27] H.-J.Sommers, A.Crisanti, H.Sompolinsky, and Y.Stein, Phys.Rev.Lett. 60, 1895 (1988); A. Edelman, J. Multivariate Anal. 60, 203 (1997) 
[28] K.B.Efetov, Phys.Rev.Lett. 79, 491 (1997); Phys. Rev. B 56, 9630 (1997)

[29] Y.V. Fyodorov 1997, in book: "Supersymmetry and trace formula in chaos and disorder", proceedings of the NATO ASI Conference, Cambridge (J.Keating, D.Khmelnitski and I.Lerner, eds.)

[30] P.A.Moldauer, Phys.Rev. v.157,907 (1967); M.Simonius Phys.Lett. v.52B, 279 (1974)

[31] S.Albeverio,F.Haake,P.Kurasov,M.Kus and P.Seba , J.Math.Phys. v.37, 4888 (1996) 


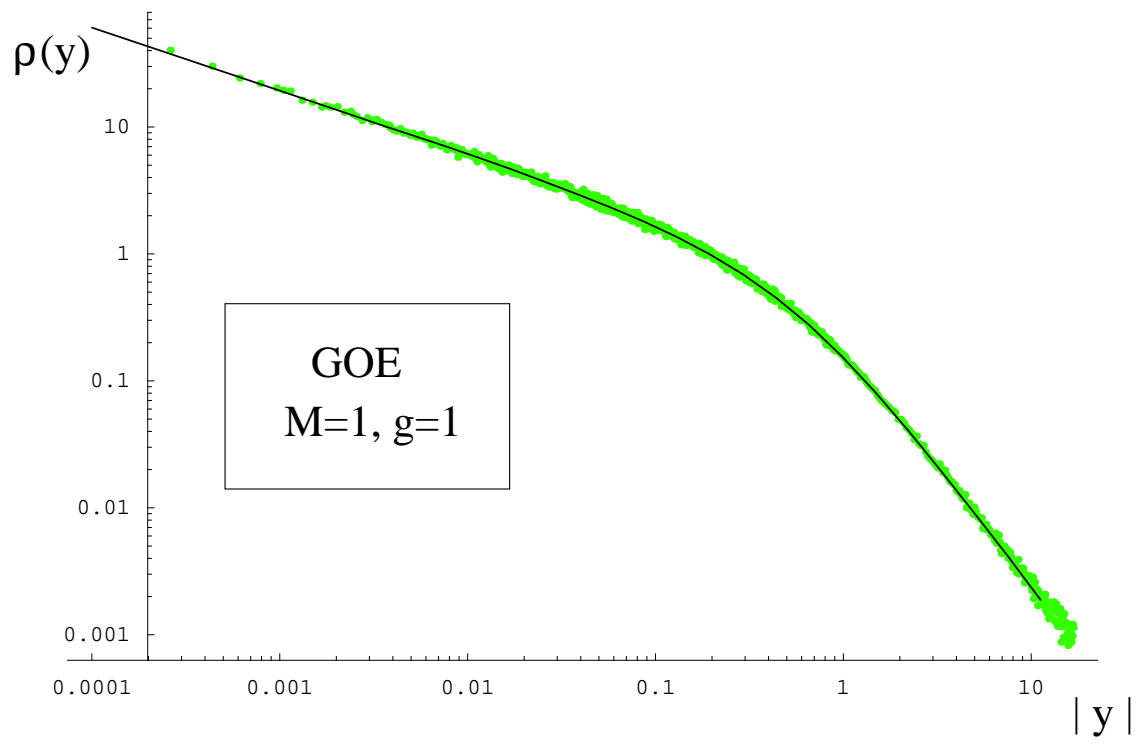

FIG. 1. Comparison between the predictions of our formula Eq.(5) for one channel case $M=1$ at "perfect coupling" $\mathrm{g}=1$ and the results of direct numerical diagonalization of $K=10000$ complex matrices of the size $400 \times 400$. 


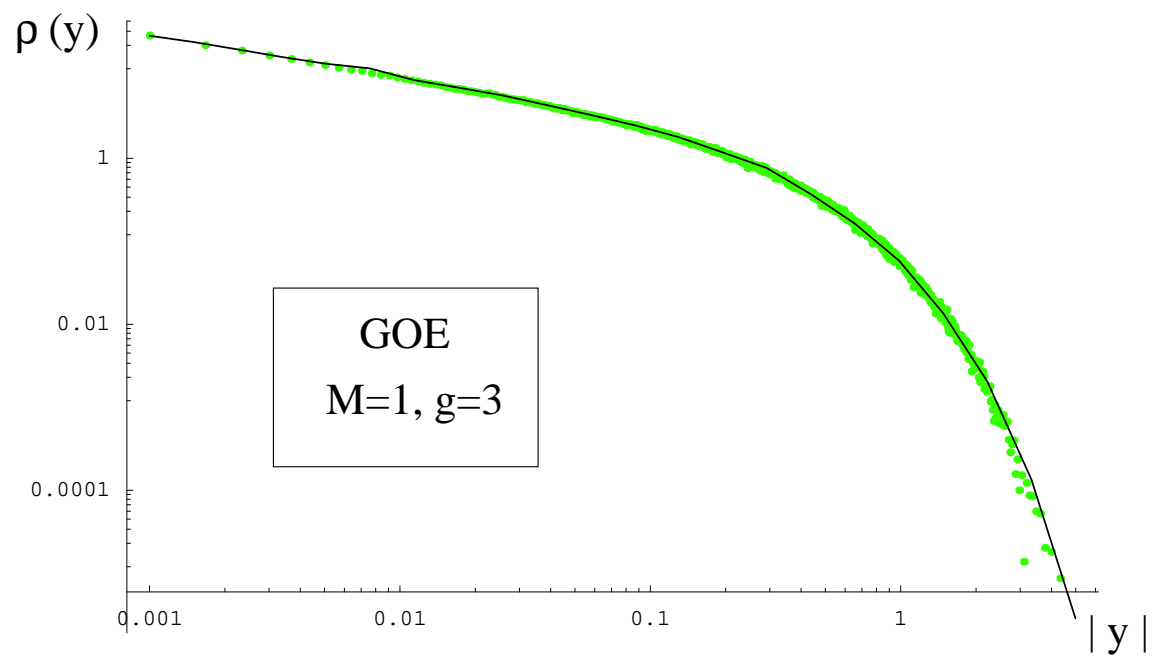

FIG. 2. Same as Fig.1 for $M=1, g=3$. 